\title{
Uncanny as Usability Obstacle
}

\author{
Angela Tinwell \\ The University of Bolton, School of Games Computing and Creative Technologies, \\ Deane Road, Bolton, UK, BL3 5AB \\ A.Tinwell@bolton.ac.uk
}

\begin{abstract}
The eerie feeling attributed to photo-realistic human-like video game characters may serve as a usability obstacle leaving viewers dissatisfied with a particular character for a video game. This study investigates the relationships between user satisfaction and perceived strangeness and between user satisfaction and human-like appearance for virtual characters. 65 participants were asked to rate 13 video clips of 12 different virtual characters and one real human. The results indicate that the Uncanny Valley does serve as a usability obstacle with a strong correlation between a user's satisfaction rating and the perceived strangeness for a character, with the characters rated the strangest being the least satisfactory. Whilst there was still a positive correlation between human-like appearance for a character with user satisfaction, this was not as significant, with stylised and anthropomorphic characters perceived to be as satisfactory or more so than those of a photo-realistic human-like appearance.
\end{abstract}

Keywords: Video Games, Uncanny Valley, Photo-realistic, Usability Obstacle.

\section{Introduction}

With increasing sophistication of movement and gesture in real-time computer character animation and with improvements in the photo-realist appearance of characters, game developers are aiming to increase player engagement and the immersive experience. However there is a technical and conceptual brick wall to be overcome that is known as the 'Uncanny Valley'. It sometimes seems that the more human-like game characters become the more vociferously potential users will object. As video games are able to approximate photo-realistic characters, will this add to the overall satisfaction for a user or act as a usability obstacle because the user becomes dissatisfied with the characters?

Masahiro Mori recognised that as a robot's appearance became more human-like it was perceived as familiar to a viewer, until finer nuances from human norms caused them to appear creepy, evoking a negative effect for the viewer. The positive relationship Mori identified between the perceived familiarity for a robot with human likeness is interrupted at certain point where the robot is perceived as more strange than familiar. This interim dip occurs at the point where the robot appears close to being human, but not fully and is referred to as the Uncanny Valley. Mori hypothesised that the Uncanny Valley would be even more exaggerated with moving characters [6].

Players appear to respond to video game characters as Mori described. Quantic Dream's tech demo, The Casting for the video game Heavy Rain was first revealed at 
the third Electronic Entertainment Expo in 2006 (E3 2006). The main character Mary Smith received criticism for being uncanny and people responded to her in a negative way [1]. A lack of synchronisation with speech and lip movements is one of the factors that people found disturbing. It was also cited that her behaviourisms and appearance are similar to that of a sociopath with characteristics such as facial asymmetries and behaviour making her appear odd evoking an eerie sensation for the viewer. Whilst many have critiqued The Casting with feedback provided in online forums and articles, this study will provide formal validation to determine whether photo-realistic characters, including Mary Smith, evoke the eerie sensation attributed to the Uncanny Valley and what impact this has on user experience.

One of the common factors with regard to user experience is the overall satisfaction for a user and how much a user enjoys interacting with an interface. Users may become dissatisfied if they become frustrated or confused with an interface or prefer another design. For the purpose of this study, satisfaction is defined as how much a user would enjoy interacting with a character within the context of a video game. The character may serve the role as a protagonist, antagonist or an opponent's avatar to which a user relates within the context of a multiplayer video game. To provide the best possible intended experience for a user, it may be recommended that game developers be aware of what impact photo-realistic human-like characters may potentially have on user experience within the context of a video game.

Recent projects by the facial animation company Image Metrics for photo-realistic characters have been acclaimed as finally overcoming the Uncanny Valley. For the photo-realistic character the Emily Project, VFXworld's Peter Plantec officially pronounced that Image Metrics has built a bridge across the Uncanny Valley [8]. By means of experiment, this study analyses case studies such as the Emily Project to assess if feelings of eeriness associated with the uncanny are relevant to photo-realistic humanlike characters and if so what impact this has for user satisfaction.

It is currently unclear as to when game developers will be able to overcome the Uncanny Valley with estimations that the computer processing power required for rendering completely convincing humans may not be available for up to another twenty years [4]. The results of this study imply that during this time, in the pursuit of photo-realism, it may be advisable for video game developers to be careful not to inadvertently create characters that will put users off. A decrease in satisfaction for the user may result in them spending less time interacting with the computer game or avoiding it all together. The game's popularity may stagnate or decrease as users become increasingly dissatisfied interacting with the video game characters.

This study builds upon the work of previous investigation into the application of Mori's theory to virtual characters including popular video game characters. Schneider et al. (2007) investigated the relationship between human-like appearance and attraction [7]. Presented with still slides for each character, participants were asked to rate their opinion on a five point scale for how human or non-human they felt the character appeared and how attractive or repulsive they felt the character to be. The results implied that making a character more human-like can increase how attractive that character is perceived to be and the safest combination for a character designer seems to be a clearly non-human appearance with the ability to emote like a human. In response to Mori's interest in how motion would impact perception, stating that the valley would be even more exaggerated with moving characters, Schneider et al. (2007) stated that another 
study using video clips instead of still images would help better support their theory. Based on these findings, for the intention of this research video clips of virtual characters have been used as stimuli for the means of experimentation.

For the horror genre of games, elements of the uncanny can serve to enhance the experience for the user, evoking feelings of tension and dread when interacting with video game characters [3]. MacDorman (2006) has stated that as well as using the principles of Mori's theory of the Uncanny Valley to create design principles that engineers can use to develop aesthetically pleasing robots, the theory can work to the advantage of engineers when designing robots with the purpose to be unnerving within an appropriate setting and context [5]. It is also noted that the perception of eeriness does not always serve as a negative impact for the viewer, with the horror game genre being such a success. However, not all computer games intend to evoke the emotions associated with a horror game for a user. Many video game characters serve the role of a protagonist within the game intended to build alliance and a sense of familiarity with the player. Players may be dissatisfied with photo-realistic human-like video game protagonists that contest the sense of the real. To build on this theory, two zombie characters were included within this study to see if the results proved that the horror genre of character would conform to Mori's theory of the Uncanny Valley.

From the standpoint of previous research regarding human emotion and the uncanny valley, Ho et al. (2008) carried out an experiment to investigate which emotion terms are related to eeriness, creepiness, strangeness and human likeness [2]. The results showed that the emotion term fear is strongly predictive of eerie and creepy for robots with disgusted, nervous and shocked also being significant, whilst relaxed predicts a high human likeness. The emotions amazed, attracted, curious, excited, fondness, and happy were regarded as more positive and less significant predictors of the terms eerie and creepy. Based on these findings participants were also asked to select from one of 14 emotion terms that best described how each video game character made them feel. The emotion terms fear, shocked and nervous, were included in the study to provide further analysis for implied levels of eeriness for each character. The emotion terms and scores attributed for each character were then listed against average satisfaction ratings to provide further evidence for implied levels of eeriness for each character.

The findings from this study might be used as a reference by game developers to ensure that characters created for a video game do not unwittingly serve as a usability obstacle for the viewer. Similarly the findings could allow game developers to consider whether photo-realistic human-like characters are necessary for a game (and what advantages there would be for the user) when a more stylised or anthropomorphic design to represent the human form may instead be beneficial. The findings will also provide an indication of whether 3D modellers are yet close to overcoming the Uncanny Valley as previously claimed for photo-realistic human-like characters.

\section{Methodology}

\subsection{Experiment: Ratings of Video Clips}

65 participants were used for this experiment with 59 males and 6 females. The participants were mainly university students from the School of Games Computing and Creative Technologies at Bolton University in addition to professionals working 
within the academic sector and video games industry. Three of the participants worked as 3D modellers from the company 3D Framework. The majority of participants had an advanced experience of playing video games (41) with 21 participants having a basic level and only three participants with no previous experience of playing video games.

Participants were presented with 13 video clips of a selection of virtual characters and one real human placed in different settings and engaged in different activities. The video clips included, six photo-realistic characters, the Emily Project and the Warrior by Image Metrics, Mary Smith from Quantic Dream's tech demo The Casting, Alex Shepard from Silent Hill Homecoming, Brucie Kibbutz from Grand Theft Auto IV, two zombies from the video games Silent Hill Homecoming (Zombie 1) and Alone in the Dark (Zombie 2), three stylised human-like characters including a Chatbot, Lara Croft and Nintendo's Super Mario, two anthropomorphised characters, Sonic the Hedgehog and a Sackboy from Little Big Planet and one real human.

Participants were asked on a web based questionnaire to rate on a nine-point scale how human-like they perceive the character to be from nonhuman-like (1) to very human-like (9) and from very strange (1) to very familiar (9). They were then asked to rate them for satisfaction on a ten-point scale, ranging from dissatisfactory (1) to very satisfactory (10). For the purposes of this study it is important to define the rating satisfaction. Participants were asked to rate how satisfactory they perceived a character to be within the context of a video game and how much they would enjoy interacting with that character within a video game. To measure how the characters made the participants feel, participants were asked to select one of 14 emotion terms. The video clips were played in random order to each participant.

\subsection{Results}

The mean satisfaction rating for characters was 6.65 with a standard deviation of 1.33 . The mean average ratings for the emotion terms were: amazed (mean=2.62 $\sigma=3.20$ ), angry (mean=1.00 $\sigma=1.41$ ), attracted (mean=2.69 $\sigma=4.35)$, confused (mean=5.00 $\sigma=3.65)$, curious (mean=8.62 $\sigma=6.10$ ), dislike (mean=5.38 $\sigma=4.65$ ), excited $($ mean=6.15 $\sigma=4.56)$, fear $($ mean=4.00 $\sigma=7.42)$, fondness $($ mean=4.00 $\sigma=4)$, happy (mean=7.69 $\sigma=9.88)$, irritated (mean=7.54 $\sigma=7.68)$, nervous (mean=3.77 $\sigma=4.64$ ), relaxed $($ mean $=5.31 \sigma=5.71)$, and shocked (mean=1.62 $\sigma=1.66)$. The characters with the highest satisfaction ratings had the highest scores for the emotion terms: amazed, attracted, curious, excited, happy, and fondness which were considered to convey a more enjoyable experience for a user. The emotion terms confused, dislike, fear, irritated, nervous and shocked were associated with a negative, unpleasant experience for the user with a lower satisfaction rating.

Table 1 shows the mean average satisfaction rating for each character and the top three emotion term scores attributed to each character. As results from experiments using robots have previously shown [2], the emotion term relaxed is also a strong indicator of human likeness for virtual characters with the real human in this study receiving the highest score for relaxed (23). For the case study the Emily Project, the results show that whilst the photo-realistic human-like character Emily scored well for emotions not associated with eeriness, such as amazed (9), curious (8), and relaxed 
Table 1. Average Satisfaction Ratings and Top Three Emotion Terms for each Character

\begin{tabular}{lcl}
\hline Character & $\begin{array}{c}\text { Mean average } \\
\text { satisfaction rating }\end{array}$ & Top three emotion term scores \\
\hline Mario & 8.4 & Happy (33), Excited (8), Fondness (7) \\
Lara Croft & 7.7 & Attracted (16), Excited (15), Curious (7) \\
Sonic & 7.6 & Happy (18), Fondness (15), Excited (11) \\
The Warrior & 7.5 & Curious (21), Nervous (12), Confused (9) \\
Emily & 7.4 & Amazed (9), Curious (8), Relaxed (8) \\
Sackboy & 7.0 & Happy (20), Curious (11), Excited (9) \\
B. Kibbitz & 6.9 & Irritated (15), Excited (12), Dislike (6) \\
Human & 6.7 & Relaxed (23), Irritated (13), Curious (9) \\
A.Shepherd & 6.7 & Curious (20), Nervous (12), Relaxed (6) \\
Zombie 2 & 6.1 & Fear (20), Dislike (11), Nervous (10) \\
Mary Smith & 5.9 & Irritated (12), Curious (9), Confused (8) \\
Zombie 1 & 5.3 & Fear (21), Confused (12), Curious (10) \\
Chatbot & 3.2 & Irritated (28), Dislike (18), Confused (5) \\
\hline
\end{tabular}

(8) the character also received an above average score for the emotion term confused (7) and shocked (5) with just below an average score for irritated (7). 8 participants rated associated this character with the emotion term relaxed, an indicator of human likeness. The results demonstrate that whilst the majority of participants (44) associated this character with emotion terms typical of a satisfactory experience, 22 participants associated this character with emotion terms typical of a less satisfactory experience.

The three most satisfactory characters received high scores for the emotion terms, happy, excited and fondness. The stylised Lara Croft received the highest score for attracted (16) with this type of character perceived to be more attractive than photorealistic human-like characters and real humans within the context of a game.

The least human-like of the two zombie characters, Zombie 1, received the highest score for the emotion term fear (21). With a less obvious human form participants felt confused as to how a character could appear so grotesque and frightening. 10 participants were curious and intrigued to learn more about this character perhaps to understand why the character's appearance is so frightening, or for those participants who enjoy horror video games seeking to gratify a curiosity in frightening themselves. The more human-like Zombie 2 received a high score for the emotion term fear (20). Despite a more obvious human-like form, 11 participants disliked the frightening appearance for this character which also made them feel nervous.

The character Mary Smith received the third highest score for fear (4), with most participants finding the character irritating (12). Participants were curious to learn more about this character; however 8 participants were still left confused by her strange and unnatural appearance. The protagonist Alex Sheppard scored just an average satisfaction rating of 6.7 and made 12 participants feel nervous with one of the highest scores for this emotion term. As the main figure within the game intended as the hero, this character may have expected to receive an above average rating for user satisfaction and higher ratings for emotion terms such as fondness and attracted. The elements of motion quality, including subtle nuances in facial animation and a lack of lip synchronisation with sound, may serve as a usability obstacle for this character which has perhaps put users off. 


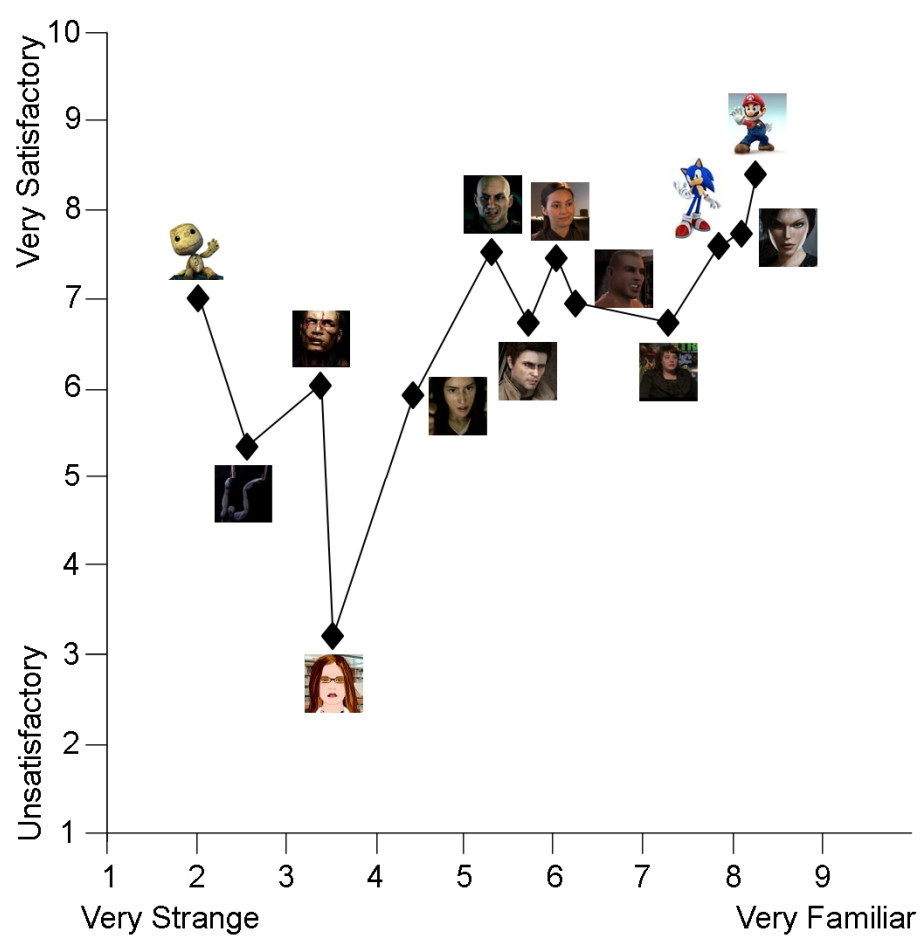

Fig. 1. The solid line plots the relationship between perceived familiarity (on the strange versus familiar scale) and satisfaction

Figure 1 plots the relationship between perceived familiarity (on the strange versus familiar scale) and user satisfaction. The results demonstrate a strong correlation between a user's satisfaction rating and the strange versus familiar rating $(r=.75)$, with characters perceived to be strangest as the least satisfactory. Figure 2 plots the relationship between how human-like a character is perceived to be (on the nonhumanlike versus very human-like scale) and user satisfaction. There was still a positive correlation between a user's satisfaction rating for how human-like a character was perceived to be, however this was considerably less $(r=.2)$. From these results it is implied that the uncanny phenomenon for virtual characters does serve as a usability obstacle with the perceived impact of the uncanny having a direct influence on a user's overall satisfaction with the character.

The results show that users found the photo-realistic human-like characters more satisfactory than a real human within the context of a video game. This may demonstrate a user's desire to escape from reality whilst playing a video game and that a real human may not be acceptable within the context of a video game.

Stylised, iconic characters such as Lara Croft and Mario and the anthropomorphic character, Sonic the Hedgehog are more familiar and subsequently more satisfactory to users than photo-realistic human-like characters. The anthropomorphic character Sackboy is less satisfactory and familiar than Sonic the Hedgehog and the photorealistic human-like characters, Emily and the Warrior. This type of character still 
exhibits some human traits such as gesture and facial expressions but is rated the least human-like out of all characters. This implies that a user demands a more sophisticated appearance for an anthropomorphic type character than the simplistic appearance of the Sackboy.

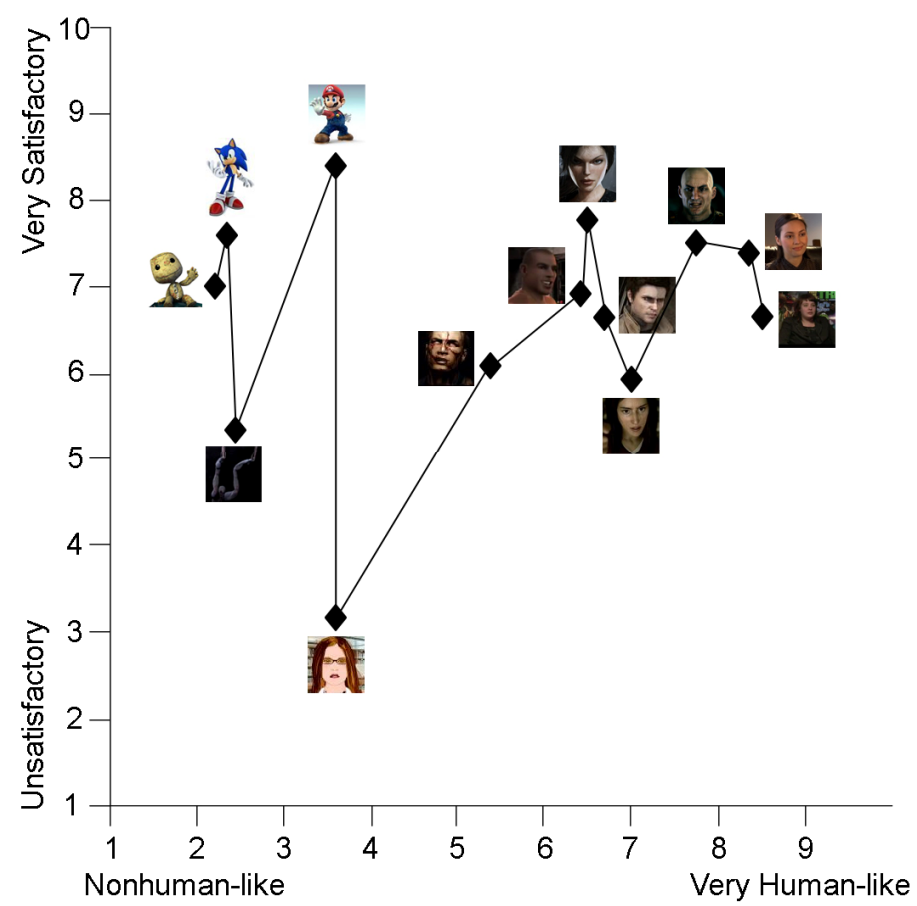

Fig. 2. The solid line plots the relationship between how human-like a character is perceived to be (on the nonhuman-like versus very human-like scale) and satisfaction

The photo-realistic character Mary Smith was rated on a similar satisfaction level as Zombie 2. The uncanny phenomenon is evident for this photo-realistic human-like character provoking the same impact as characters intended for a horror game.

The stylised Chatbot character was perceived as unsatisfactory within the context of a video game. The sound from the Chatbot was regarded as the most unsatisfactory characteristic. The Chatbot was regarded as the most irritating with 28 participants attributing the emotion term irritated for this character, whilst 18 participants took a firm dislike to the Chatbot (see Table 1). This result shows that users expect more than the simplicity of the Chatbot's appearance for a video game, with the lack of lip synchronisation with speech being the most irritating characteristic.

Figure 3 plots the mean average ratings for how human-like a character is perceived to be against perceived familiarity and reveals more than one single valley in the plot. Horror game characters intended to contest the sense of the real do conform to a U-shaped valley. The photo-realistic character Emily is perceived to be very human-like and may be close to overcoming the Uncanny Valley phenomenon as 


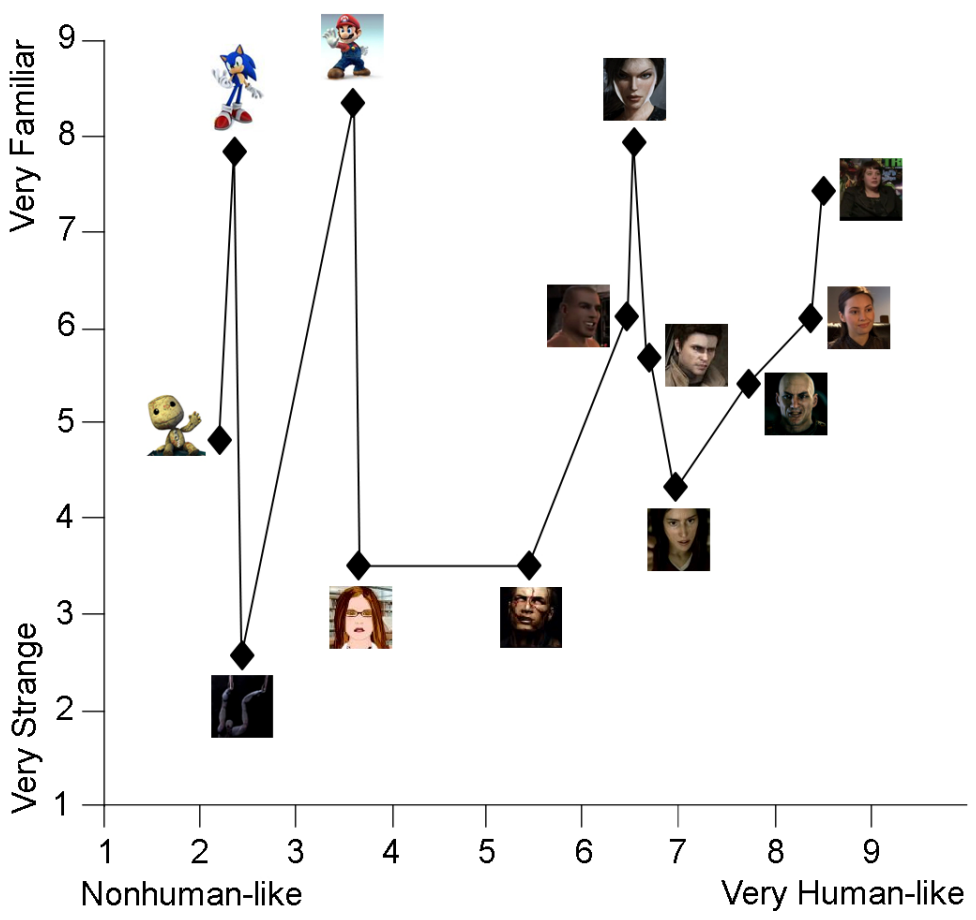

Fig. 3. The solid line plots the mean average ratings for how human-like a character is perceived to be on the nonhuman-like versus very human-like scale and perceived familiarity on the strange versus familiar scale

proclaimed by Plantec [8]. The photo-realistic characters Mary Smith and Alex Shepherd that are placed close to or within a dip may provoke an impact of the uncanny and put users off using a game when they are otherwise not intended to do so. The Chatbot character is placed within a valley and is perceived to be less human-like and as strange as the Zombie 2 character. Sonic the Hedgehog and Mario are widely accepted as familiar, with little risk of evoking the eeriness attributed to the uncanny, despite their nonhuman-like appearance. The stylised character Lara Croft is perceived to be not as eerie as all of the photo-realistic human-like characters, despite being rated less human-like than four of the photo-realistic human-like characters.

\section{Discussion}

The results suggest that the eerie feeling attributed to video game characters does serve as a usability obstacle leaving viewers dissatisfied with photo-realistic humanlike characters that appear strange as opposed to familiar. For a user to find a video game character satisfactory within the context of a game it is not necessary for the character to have a photo-realistic human-like appearance. A character should evoke a greater sense of familiarity as opposed to eeriness unless they are intended to evoke fear such as a zombie for the horror game genre. 
With real humans perceived as less satisfactory than animated virtual 3D characters within the context of a video game, the pursuit for photo-realistic human-like characters is worth the endeavour, but until video game developers have the sufficient technology and expertise to overcome the uncanny, stylised or anthropomorphic characters that exhibit human traits are more satisfactory for a user.

This study showed that users respond to uncanny photo-realistic human-like characters in the same way they would respond to zombies intended for horror games. If photo-realistic characters are to be used as protagonists within a game then it is advisable that they should be tested to ensure that there is a sense of familiarity for the user and that game developers have not unwittingly put users off the character. Contrary, elements of the uncanny may be exaggerated for horror game antagonist characters to provoke a greater sense of eeriness and fear for the user. Elspeth Tory, the Animation Project Manager for Ubisoft's video game Assassin's Creed has acknowledged that in order to combat the uncanny it is essential that games developers do not work on their games in isolation as then they are purely relying on their own sense of aesthetics to make a character believable [1]. If photo-realistic human-like characters are to be included within a video game, it may be worthwhile for game developers to carry out testing within the development stages of a game specifically to rate a user's satisfaction for the characters within the context of a video game. This type of testing will help to ensure that risk of the uncanny for characters is reduced and users will not be inadvertently put off the video game.

Creating overly-simplistic characters for video games is not advisable as most users found them unsatisfactory within the context of a game. Users expect more than the simplistic appearance for a Chabot character (disliked and the most irritating). The jerky motion exaggerated the lack of lip synchronisation with speech for this character. The simplistic nature of the Chatbot for both image and sound annoyed and confused users, with the Chatbot rated as strange as a zombie character.

The Emily Project is perceived to be very human-like and this study showed that it is close to overcoming the Uncanny Valley as proclaimed by Plantec [8]. Most users rated this character as satisfactory, however those working within the industry and with a high level of gaming experience were more scrupulous in detecting the finer nuances of facial animation that risk the character being uncanny. The reflectivity of the eyes, the texture of the skin surface and lack of movement in facial muscles on the forehead, mouth and eyes were noted during the experiment as elements that made this character seem less believable. Emotion terms used to describe this character included confused, shocked, fear and nervous demonstrating that this character does evoke the eeriness attributed to the uncanny for some users. The results from this study may provide support to prove that Emily is close to overcoming the Uncanny Valley however it is evident that there are subtle elements of motion quality that can still be improved to increase the character's believability.

In future studies the methodology for testing this hypothesis could be improved by rating the experience and expertise of participants in 3D modelling to provide a more robust hypothesis as to whether the Emily Project and other photo-realistic humanlike characters may be close to overcoming the Uncanny Valley phenomenon.

Not all characters featured within the study had sound. For those characters with sound this aspect was shown to be a contributing factor in the ratings for user satisfaction and strangeness as well as visual appearance. There is a tremendous potential for 
future research in this area to study how motion qualities such as facial expression, timing, contingency during interaction and sound contribute to the feeling that a virtual character is uncanny. Defining an initial conceptual framework for the fearevoking aspects of motion quality that exaggerate the uncanny may also be used by game developers seeking to increase the fear factor for characters in horror games.

\section{References}

1. Mori, M.: Bukimi No Tani [The Uncanny Valley]. Energy 7(4), 33-35 (1970)

2. Gouskos, C.: The Depths of the Uncanny Valley (July 8, 2006) http://uk.gamespot.com/features /6153667/index.html

3. Weschler, L.: Why is This Man Smiling? Wired Magazine 10(06), 16-17 (2002)

4. Schneider, E., Wang, Y., Yang, S.: Exploring the Uncanny Valley with Japanese Video Game Characters. In: Situated Play, DiGRA 2007 Conference, pp. 546-549 (2007)

5. Plantec, P.: The Digital Eye: Image Metrics Attempts to Leap the Uncanny Valley (August 7, 2008), http: / / vfxworld. com / ?atype=articles\&id=3723\&page =1

6. Hoeger, L., Huber, W.: Ghostly manipulation: Fatal Frame II and the Videogame Uncanny. In: Situated Play, DiGRA 2007 Conference, pp. 152-156 (2007)

7. MacDorman, K.: Subjective ratings of robot video clips for human likeness, familiarity, and eeriness: An exploration of the uncanny valley. In: ICCS/CogSci-2006 Long Symposium: Toward Social Mechanisms of Android Science, Vancouver, Canada (2006)

8. HO, C.-C., Macdorman, K., Pramono, Z.A.D.: Human emotion and the uncanny valley: A GLM, MDS, and ISOMAP analysis of robot video ratings. In: The Third ACM/IEEE International Conference on Human-Robot Interaction, Amsterdam, pp. 1148-1158 (2008) 